\title{
Neoadjuvant Chemotherapy in Lung Cancer: Our Results after Twelve Years of Surgical Resection
}

\author{
Hazem $Z^{1}$, Ahmed $A^{1}{ }^{1 *}$, Abderrahmen $A^{1}$, Mahdi $A^{1}$, Sonia $O^{2}$, Hanen $A^{3}$ and Adel $\mathbf{M}^{1}$ \\ ${ }^{1}$ Department of Thoracic Surgery, Abderrahmen Mami University Hospital, Ariana, Tunisia \\ ${ }^{2}$ Department of Anesthesia and Reanimation, Abderrahmen Mami University Hospital, Ariana, Tunisia
}

${ }^{3}$ Department of Ibn Nafiss Pneumology, Abderrahmen Mami University Hospital, Ariana, Tunisia

\begin{abstract}
Introduction: Lung cancer is characterized by its frequency and prognosis, which remains reserved by the fact that the diagnosis is often made at the locally evolved or metastatic stages. The aim of this study is to evaluate the contribution of neoadjuvant chemotherapy to the resectability of non-small cell lung carcinoma, while highlighting its impact on the incidence of surgical complications and the effect of this therapeutic strategy on survival.

Materials: We have carried out a retrospective analysis of patients who underwent surgical treatment of nonsmall cell lung cancer (NSCLC) after neoadjuvant chemotherapy (NAC), between March 2000 and October 2012 at the Thoracic Surgery Department of Abderrahmen MAMI University Hospital.

Results: During the study period, 103 patients were referred to our institution for NSCLC's curative surgery after a NAC. Ninety five (95) patients are operated and 8 were not because of tumor progression after NAC or a high risk of operative mortality. The evaluation after the NAC showed a partial response in 77 cases $(75 \%)$. More than half patients (57 cases: $55.7 \%$ ) had a down-staging after the NAC. Male gender, and age increase postoperative complications significantly $(p=0.042)$. Global survival was calculated for 87 patients $85 \%, 56 \%, 50 \%$ respectively at 1 year, 2 years and 5 years. The mean survival was 25 (range: 2 to 132) months. Global survival was increased significantly with T1 status $(p=0.003)$ and M0 status $(p<0.005)$ before NAC and also after NAC $(p=0.032)$. Tumor localization had a significant impact on survival $(p=0.034)$ with best survival with tumor in middle lobe and worst survival with bilobar tumors.

Conclusion: We noticed an increased survival with age $<60$ years, feminine gender, well differentiated tumor, patient with no parietal involvement, NAC protocol other than Gemzar-Cisplatine, complete or partial response to NAC, pneumonectomy as the type of resection, no enlargement resection, uneventful postoperative, R0 resection, NO status after NAC and finally pNO status.
\end{abstract}

Keywords: Non-small cell lung carcinoma; Neoadjuvant chemotherapy; Thoracic surgery

Abbreviations: NSCLC: Non-Small Cell Lung Carcinoma; NAC: Neoadjuvant Chemotherapy; IASLC: International Association for the Study of Lung Cancer; ATS: American Thoracic Society; ERS: European Respiratory Society

\section{Background}

Lung cancer is characterized by its frequency and prognosis, which remains reserved despite the improvement of therapeutic management. Non-small cell lung carcinomas (NSCLC) are $80 \%-85 \%$ of lung cancers [1]. The pejorative prognosis of this cancer can be explained by the fact that the diagnosis is often made at the locally evolved or metastatic stages. Indeed, $90 \%$ of NSCLCs are discovered at these stages [2]; $30 \%$ are found in stage III, of which one third is classified as IIIA, considered as potentially resectable and two thirds are classified as IIIB, considered as a non-resectable disease except in special cases.

A Neoadjuvant treatment is therefore necessary to reduce the tumor volume (down-staging), leading to the resectability of tumors initially unresectable [3]. Neo-adjuvant chemotherapy aims not only to make possible, or to optimize, the resection of unresectable tumors from the outset or at the limit of resectability, but also to increase survival by acting early on micro-metastasis [4].

The aim of this study is to evaluate the contribution of neoadjuvant chemotherapy to the resectability of NSCLC, while highlighting its impact on the incidence of surgical complications and the effect of this therapeutic strategy on survival [5].

\section{Materials and Method}

We have carried out a retrospective analysis of patients who underwent surgical treatment of non-small cell lung cancer (NSCLC) after neoadjuvant chemotherapy (NAC), between March 2000 and October 2012 at the Thoracic Surgery Department of Abderrahmen MAMI University Hospital.

\section{Recruitment}

We included all patients, who had histopathological diagnosis of NSCLC and were operated after a NAC, combined or not to another therapy, with a curative intent. We have excluded patients operated with a palliative or a diagnostic intents.

Eligibility of NAC was based on: 1) locally advanced tumor (stage IIIA and selected stage IIIB) considered as potentially resectable; 2) and expected benefit of this chemotherapy is down-staging and R0 resection. Eligibility of surgery for NSCLC was based on: 1) accepted

*Corresponding author: Ben Ayed Ahmed, Department of Thoracic Surgery Abderrahmen Mami University Hospital, Ariana, Tunisia, Tel: +21671821182 E-mail: ahmed.benayed.tunisia@gmail.com

Received June 21, 2018; Accepted July 06, 2018; Published July 13, 2018

Citation: Hazem Z, Ahmed AB, Abderrahmen A, Mahdi A, Sonia O, et al. (2018) Neoadjuvant Chemotherapy in Lung Cancer: Our Results after Twelve Years of Surgical Resection. J Pulm Respir Med 8: 463. doi: 10.4172/2161-105X.1000463

Copyright: ( 2018 Hazem Z, et al. This is an open-access article distributed under the terms of the Creative Commons Attribution License, which permits unrestricted use, distribution, and reproduction in any medium, provided the original author and source are credited. 
morbidity and operative mortality estimation as defined by consensus; 2) sufficient pulmonary reserve after the surgery (according to classic criteria of pulmonary resection); 3) and a complete resection (R0) considered as possible. All decisions were taken by multidisciplinary meetings, including thoracic surgeons, oncologists, radiologists, and radiotherapists.

Tumors were staged based on clinical information, bronchial fibroscopy and other imaging techniques (indicated and used as defined by consensus). Before initiating the NAC, the histopathological diagnosis was confirmed by a biopsy (percutaneous or through other technique). The chemotherapy protocol comprises 2 to 4 cycles of a combined therapy based on cisplatin.

The evaluation of NAC was achieved by clinical examination, bronchial fibroscopy, thoraco-abdomino-pelvic CT scan performed 2 to 3 weeks after the end of the NAC according to RECIST criteria.

The Follow-up was performed in the Thoracic Surgery Department and the Oncology Unit in our institution. The endpoint date was set on October 31, 2013. Global survival was calculated from the date of radical surgery up to the endpoint date. We excluded from the calculation perioperative deaths and patients with exploratory thoracotomy.

\section{Recorded data}

The following parameters were recorded:1) characteristics of patients: age (at the time of surgery), gender, antecedents, general state; 2) diagnosis and staging: location, histological type (WHO 2004 classification but since 2011 adenocarcinoma tumors were classified according to IASLC, ATS, ERS), differentiation grade, TNM status and stage ( $7^{\text {th }}$ edition of IASLC applied before NAC); 3) NAC: delay between diagnosis and NAC, chemotherapy protocol (drugs, cycles number, association to a neoadjuvant radiotherapy (NAR)), evaluation of NAC (response, TNM and Down-staging), treatment of metastasis; 4) Surgery and histopathology: delay between NAC and surgery, peroperative constitutions, type of resection, postoperative complications, resection quality, pTNM status and down-staging, 5) Postoperative follow up: delay between surgery and first consultation, relapse (delay, location, treatment), survival.

\section{Statistical analysis}

Statistical analysis was performed by using SPSS $^{\circledR}$ software version 20 (SPSS Ink, Chicago, IL, USA).

Numerical results were expressed on average if the variable distribution was Gaussian and on median if it were not. We used the Student's t-test for the comparison of means and the standard deviation for the comparison of percentages after verification of applicability conditions. Estimation of survival was performed according to Kaplan and Meier method. Survival data were compared using the Log-Rank test for a single variable and the Cox model for the multivariate study. The difference was considered significant if $\mathrm{p}<0.05$.

\section{Results}

During the study period, 103 patients were referred to our institution for NSCLC's curative surgery after a NAC. Ninety five (95) patients are operated and 8 were not because of tumor progression after NAC or a high risk of operative mortality. The median age was 55.5 (range: 74 to 25) years and the sex ratio was 13.7 (males: 96 versus females: 7).

In 64 cases $(62 \%)$ tumor located at right lung and in 38 cases (36.9\%) in the upper right lobe. The histopathological type was an adenocarcinoma in 44 cases (42.7\%) and well differentiated in 37 cases

\section{(35.9\%).}

Primitive tumor was classified T3 in cases 33 cases (32\%), N1 in 62 cases (60\%) and M0 in 81 cases (78.6\%). Thus, 50 cases (48.5) were classified IIIA.

\section{Neoadjuvant chemotherapy}

The median delay between diagnosis and NAC was 5 (range: 1-32) weeks. Gemzar-Cisplatine was used in 66 cases $(64 \%)$. The median of Cycles number was 3 (range: 1-8) cycles. Five patients had a second line chemotherapy and 6 patients had a NAR (concurrent in 3 cases). The evaluation after the NAC showed a partial response in 77 cases $(75 \%)$. More than half patients (57 cases: $55.7 \%$ ) had a down-staging after the NAC but we notice that all metastatic patients (intra or extrathoracic) kept the same M status after the NAC even if some metastasis were completely disappeared.

\section{Surgery and histopathology}

The median delay between NAC and surgery was 6 (range: 52-4) weeks. The median tumor size was $3.5 \mathrm{~cm}$, with a maximum of $13 \mathrm{~cm}$. In two cases the tumor was completely sterilized by chemotherapy. We noticed a loco-regional encroachment in 31 cases (30\%) with 5 cases where a radical resection was impossible. Fifty three (51\%) patients underwent a lobectomy and 29 patients (28\%) underwent a pneumonectomy. Resection was associated with a loco-regional enlargement in 22 cases. The postoperative was uneventful in 61 cases (64.2\%). The postoperative mortality and morbidity was $3.15 \%$ and $35.74 \%$ respectively. We noticed that the type of resection, patient antecedents $(p=0.673)$, general state $(p=0.929)$ and loco-regional enlargement $(\mathrm{p}=0.092)$ had no significant impact on postoperative morbidity, while Male gender, and age increase postoperative complications significantly $(\mathrm{p}=0.042)$.

Resection was $\mathrm{R} 0$ in 80 cases $(84.2 \%)$. Considering the pTNM status, there was a down-staging compared to the initial status of the disease in 65 cases (63\%). The down-staging was more important with T4 status, N2 status, squamous cell carcinoma (SCC) histopathological type, well differentiated tumor and Gemzar-Cisplatine NAC protocol.

\section{Postoperative follow up and survival}

The median delay between surgery and first consultation was 6 weeks. The relapse rate after radical resection was $24.3 \%$ (25 patients). The median delay between radical resection and relapse was 9.5 (range: 2 to 40) months. Ten patients had chemotherapy as a treatment of relapse.

Global survival was calculated for 87 patients $85 \%, 56 \%, 50 \%$ respectively at 1 year, 2 years and 5 years. The mean survival was 25 (range: 2 to 132 ) months. Global survival was increased significantly with $\mathrm{T} 1$ status $(\mathrm{p}=0.003)$ and M0 status $(\mathrm{p}<0.005)$ before NAC and also after NAC ( $\mathrm{p}=0.032)$. Thus, the lowest survival was noticed in Stage VI. Survival is inversely proportional to the pTNMstage with a significant difference $(p=0.029)$. Tumor localization had a significant impact on survival ( $\mathrm{p}=0.034)$ with best survival with tumor in middle lobe and worst survival with bilobar tumors.

Age $(p=0.06)$, gender $(p=0.91)$, histopathological type $(p=0.26)$, differentiation grade $(\mathrm{p}=0.51)$, parietal involvement $(\mathrm{p}=0.4)$, NAC protocol and response $(\mathrm{p}=0.11)$, type of resection $(\mathrm{p}=0.95)$, enlargement resection $(\mathrm{p}=0.95)$, postoperative complications $(\mathrm{p}=0.493)$, resection quality ( $\mathrm{p}=0.76), \mathrm{N}$ status after NAC ( $\mathrm{p}=0.34)$ had no significant impact on survival. But we noticed an increased survival with age $<60$ years, feminine gender, well differentiated tumor, patient with no parietal 
involvement, NAC protocol other than Gemzar-Cisplatine, complete or partial response to NAC, pneumonectomy as the type of resection, no enlargement resection, uneventful postoperative, R0 resection, N0 status after NAC and finally pN0 status.

\section{Discussion}

The NSCLC remains a major health problem, with overall 5-year survival at all stages of $17.1 \%$ [6]. Among NSCLC cases, $30 \%$ are locally advanced tumors (stage III) [7]. Multimodal treatment has become widely used in clinical practice with curative intent for Stage IIIA [8]. Patients classified in stages I and II can be effectively treated by resection of one or more lobes, in which the tumor is localized [9]. Some of these patients (stage IB and II) receive adjuvant chemotherapy based on platinum salt. Stages III constitute heterogeneous lesions making it difficult to establish a well-defined therapeutic strategy. Stage IV patients receive chemotherapy with or without targeted therapy depending on the presence of certain gene mutations [10]. Stages IV oligometastatic can receive treatment with curative intent if associated with a response to chemotherapy. Management of these patients should be discussed on a case-by-case basis in multidisciplinary meetings.

\section{Staging and histopathology}

$\mathrm{T}$ status is an important prognosis factor [11]. The 5-year survival of a tumor whose size is $<3 \mathrm{~cm}$ is $64 \%$ versus $46 \%$ for tumors whose size is $>3 \mathrm{~cm}$ [12]. Recent studies have found that tumors with a size between 0 and $2 \mathrm{~cm}$ have a better prognosis than those with a size between 2 and $3 \mathrm{~cm}$ [13]. Morgensztern demonstrated recently that tumor size is an independent prognostic factor for overall survival in Stage III [14]; patients whose tumor is classified as IIIA with a size over $7 \mathrm{~cm}$ are $18 \%$ more likely to die from their disease than those with a tumor size between 5.1 and $7 \mathrm{~cm}$ for the same stage. The chest wall involvement is often accompanied by a lymph node involvement which makes the incrimination of the influence of the invasion of the wall on the prognosis difficult. But in this case the main predictive factors affecting long-term survival are: $\mathrm{N}$ status, complete resection and depth of thoracic wall infiltration [15]. Overall survival does not exceed 24 months when the wall is invaded after CNA versus $60 \%$ in case of non-invaded wall. The tumors classified $\mathrm{T} 4$ with vascular invasion have a poor prognosis [16]. But a few cases of prolonged survival have been reported. Inoue et al. reported the case of a patient with a tumor invading the superior vena cava, who lived for 5 years and 4 months after surgical treatment [17]. Fukuse et al. showed that patients with aortic invasion had a better survival rate compared to those with left atrial invasion [18]. Nodes invasion in the presence of a T4 tumor is an important prognostic factor. Martini et al. reported that there was no long-term survivor in patients classified T4N2, so surgery is indicated only for tumors classified as T4N0 and T4N1 [16]. In a study of 12 patients with vertebral invasion treated at the Institut Montsouris in Paris, the survival rate at 3 years was 35\% [19]. In our series, 2 patients had vertebral invasion. They both had a radical resection of the tumor and of the spinal extension. Régnard et al. concluded that the surgical treatment of NSLC with carina invasion yield acceptable results in terms of operative mortality and long-term survival, and that would be closely related to $\mathrm{N}$ status. For this type of invasion, the patients must be carefully selected. In our series, after NAC treatment and then surgery, the best survival was found in T2b patients followed by T1a and then T3. This difference is statistically significant $(p=0.032)$. The tumor infiltrated mediastinal structures in $17.5 \%$ of cases before NAC (18 patients). The survival of tumors classified $\mathrm{T} 4$ before treatment does not exceed 25 months. The 5 -year survival of T1, T2 and T3 tumors was $78 \%, 50 \%$ and $35 \%$, respectively.
The $\mathrm{N}$ status of tumor is an important prognostic factor. In the operable NSCLC, subgroups of $\mathrm{N}$ have been identified [20]. Martini found a significant 5-year survival difference between single N1 involvement and multiple N1 involvement (45\% versus 31\%) [21]. Also, the prognosis of tumors with multiple $\mathrm{N} 1$ is similar to that of $\mathrm{N} 2$ tumors [22]. For tumors classified N1, the prognosis of tumors where lymph extension is restricted to stations $14,13,12$ or 11 is better than that of tumors with station 10 , and the 5-year survival of N1 tumors with involvement of station 10 is $35.1 \%$ compared with $58.6 \%$ in the absence of this station [22]. It was shown that T1-3 N2 status, with undercarinal nodes involvement was associated with a poorer prognosis with no 5 -year survival compared to $29.5 \%$ in the absence of under-carinal nodes $(\mathrm{P}=0.0005)$. Furthermore, in a study of a group of patients with N2 lymph node involvement, different authors showed a significant 5-year survival difference between patients with multiple N2 involvement and those with single N2 involvement with 5 -year survival respectively from 9 to $23 \%$ compared with 25 to $60 \%$. There is also a difference between a Bulky-N2 and limited or discrete lymph node involvement. The survival of N2 status is also influenced by the localization of the primary tumor. Thus, Ichinose and Casali found better survival for patients with a tumor of the upper lobe (right or left) with N2 lymph node invasion [23-25]. A contralateral or supraclavicular mediastinal lymph node involvement (N3) causes the tumor to progress to stage IIIB with a 2 -year survival of $11 \%$, regardless of the tumor size. Tumors with invasion of contralateral mediastinal lymph nodes (N3) have been considered by most teams as a contraindication to curative surgery and have been associated with a zero healing rate, but some surgeons continue to suggest resection [26,27]. The proportion of N2 before NAC in our study was $60 \%$. This proportion was $16 \%$ at histology. In our series, only one patient classified N3, treated by resection of invasive supraclavicular adenopathy followed by CNA followed by surgery remained alive until the endpoint date (overall survival at 11 months). The 2-year survival of $\mathrm{pN} 2$ was $30 \%$, whereas that of $\mathrm{pN} 0$ was $60 \%$.

The presence of a metastasis marks the transition from localized disease to a systemic form of poor prognosis [27].

A single metastasis can be resected with a curative intent if the primary tumor can be completely resected but the presence of invaded lymph nodesis a factor of poor prognosis and contraindicates surgery. In our series, metastases were found in $21.4 \%$ of cases (22 patients), $50 \%$ were classified M1a and $50 \% \mathrm{M} 1 \mathrm{~b}$. If there is a single operable cerebral metastasis and the primary lesion can be controlled, resection surgery should be proposed. The survival at 5 years can then reach $21.4 \%$. Cerebral radiotherapy after metastasis resection would reduce the risk of recurrence at this level $[28,29]$. Unique cerebral metastases led to the discovery of the diagnosis in 5 patients included in our study (5\%). These lesions were treated by surgical excision in all these cases, followed by a cerebral irradiation reported in 3 of them. The chemotherapy was then carried out followed by radical surgery before complete cerebral remission. Survival in our series was not significantly affected in metastatic cases $(\mathrm{P}=0.268)$. Five-year survival was $64 \%$ for M0 patients and $46 \%$ for M1 patients. Survival is better when the primary cancer is an adenocarcinoma and if there is no lymph node involvement and depended on 4 factors including PS, sex, age, and initial NSE value according to Jacot et al. [30,31]. For hepatic metastases, radical surgery has been proposed in many patients with acceptable survival whenever the primitive was deemed resectable. For adrenal metastases, surgery can sometimes be useful, with curative intent when the primary lesion is controlled and when its stage is not advanced [32]. Finally, for pulmonary metastasis, surgery remains indicated if the primitive is not very advanced especially in the absence of lymph 
node involvement. Moreover, when it is unique, a synchronous cancer must be evoked. It is estimated that there are $4 \%$ of multiple cancers: $60 \%$ are metachronous, $40 \%$ are synchronous [32]. In our study, the overall survival of metastatic patients treated for curative intent did not reach 2 years. Five patients had recurrence. The recidivism could not be confirmed for the other cases.

Several recent European and American studies, showed a gradual decrease in squamous cell carcinoma in favor of adenocarcinoma [33]. In our series, we observed $42.7 \%$ of adenocarcinomas and $36.9 \%$ of squamous cell carcinomas. Numerous publications have studied the prognostic value of the histological type. However, the pejorative value given by some of these studies to SCC and by others to adenocarcinoma maintains controversary [34]. In our study, by comparing the two main histological types encountered, squamous cell carcinomas and adenocarcinomas, the survival difference was not significant $(\mathrm{p}=0.26)$.

\section{Neoadjuvant chemotherapy}

Consequences of preoperative chemotherapy and radiotherapy remain debated [35]. Improved survival in patients with locally advanced tumors following induction therapy has been demonstrated by several studies with an effect on control of Disease [36]. But the NAC's level of evidence is based on phase III-trials with reduced number of patients [37].

After NAC, the mediastinal down-staging was $17 \%$ and $27 \%$ respectively. The complete response was $0 \%$ and $2 \%$ [38]. In our series, and considering the pTNM stage, we noticed that there was a downstaging compared to the initial stage of the disease in $63 \%$ of the cases.

A meta-analysis of 13 controlled and randomized trials including 3224 patients demonstrated survival benefit $(p=0.001)$ in patients treated with CNA and surgery compared with those treated with surgery alone [39]. Moreover, the best treatment remains uncertain due to the heterogeneity of the treatments received and the population in these trials. NAC had potential advantages compared to adjuvant chemotherapy such as decreasing tumor size, increasing resectability and earlier eradication of micro-metastases [5]. However, NAC may delay the time of surgery; the tumor could become unresectable and progress under chemotherapy. In our series, $13 \%$ of patients remained stable and $6 \%$ progressed under NAC. Two patients out of 95 treated with NAC were unable to benefit from surgery due to a progression of their disease.

The management of $\mathrm{N} 2$ tumors is widely discussed. For example, in the recently updated US recommendations, N2 diagnosed preoperatively should benefit from multi-modal treatment that can only include surgery in prospective trials [40].

A significant gain in survival can be obtained in patients with NSCLC that can be resected immediately by cisplatin-based chemotherapy, whether neoadjuvant or adjuvant [41]. In our series, the most used protocol was gemzar-cisplatin with $68 \%$, followed by the navelbinecisplatin protocol: $14 \%$. Lower survival was found in patients treated with gemzar-cisplatin compared with other NAC protocols $(\mathrm{p}=0.11)$.

Studies have shownimproved survival of patients treated with Pemetrexed for non-squamous cell carcinomas compared to squamous cell carcinomas [42]. Hirsch et al. in their review of the literature, have demonstrated that no histopathological subtype of NSCLC has been associated with improvement or impairment of prognosis in advanced stages [43]. In our series, down-staging was more important for squamous cell carcinomas than for adenocerciomas (61\% versus $43 \%$ ).

Pisters reported that the proportion of patients who achieved a complete histological response (CHR) after NAC was 12\% (9/27), unlike Roth who reported a $0 \%(0 / 28)$ rate and Rosell who reported a rate of $3 \%(1 / 30)$ [44]. Pisters showed that 5-year survival in stage III patients with CHR was 54\% [44]. In our study, we had 14\% (13 patients) of complete histological response to the tumor and lymph nodes (pT0N0): six of them were still alive, four were lost to follow-up and three died. Five-year survival in these patients was 51\%.

\section{Surgical resection}

Among all therapeutic means currently available, complete surgical resection of the primary tumor associated with lymph node dissection stills the only treatment which offers a significant chance of curing bronchial cancer.

The guidelines of the "American College of Chest Physicians" of 2013 recommend minimally invasive pathways for small tumors without ganglionic involvement [45]. In our series, and given the advanced stage of the disease, the approach used was the posterolateral thoracotomy.

Okada et al. showed that tumor size is an important prognostic factor on which the choice of surgical technique depends [46]. Indeed, the 5-year survival rate for a group of patients with a stage I NSCLC with a tumor size of less than $20 \mathrm{~mm}$ is $96.7 \%$ after a segmentectomy, $92.4 \%$ after a lobectomy and $85.7 \%$ after a wedge resection. They concluded that the difference in survival was not significant. Wedge resection is mostly performed in patients with reduced lung capacity. In our series, the most frequent procedure was lobectomy (53 cases), followed by pneumonectomy ( 29 cases), bilobectomy ( 3 cases), Wedge resection (2 cases) and lobectomy Associated with a Wedge resection (2 cases). The resection was extended to other structures in 26 patients. Enlargement concerned the coasts in 17 cases, the rachis and the ribs in 2 cases and the left atrium in 3 cases. Patients who had no enlargement had better survival: $58 \%$ versus $47 \%$ at 2 years $(\mathrm{p}=0.95)$.

Wu et al. they showed that complete lymph node dissection had a prognostic impact on survival after resection of lung cancer [47]. In our series, lymph node dissection was associated with pulmonary resection for all patients treated with curative surgery [48].

According to Kim et al. (study of 233 patients operated after neoadjuvant therapy), there was $6 \%$ early mortality and $13 \%$ delayed mortality after pneumonectomy compared to $1 \%$ and $4 \%$ respectively for lobectomy [36]. According to Martini et al. the right seat of pneumonectomy $(\mathrm{p}=0.002)$, peroperative hemorrhage $(\mathrm{p}=0.001)$ and FEV1 before surgery $(\mathrm{p}=0.001)$ are significant predictors of postoperative complications $[48,49]$. In our study, postoperative complications were observed in 34 patients (35.8\%). The most frequent complications were atelectasis and pneumonic infections (20.6\%), followed by prolonged aerial leakage (17.6\%) and cardiac arrhythmias (14.7\%). The three postoperative deaths were related respectively to: pulmonary embolism, septic shock and ARDS. Only the age had a statistically significant relationship with the occurrence of postoperative complications. A positive relationship exists between age over 60 years and the occurrence of these complications $(p=0.042)$. Complications were more frequent after bilobectomy, followed by Wedge resection followed by lobectomy. Pneumonectomy was complicated in $34.5 \%$ of cases. Survival is better for patients with uneventful postoperative $(\mathrm{P}=0.493)$. We concluded that NAC did not increase the frequency of postoperative complications.

\section{Perspectives}

Based on the various prospective and retrospective studies, there 
Citation: Hazem Z, Ahmed AB, Abderrahmen A, Mahdi A, Sonia O, et al. (2018) Neoadjuvant Chemotherapy in Lung Cancer: Our Results after Twelve Years of Surgical Resection. J Pulm Respir Med 8: 463. doi: 10.4172/2161-105X.1000463

Page 5 of 6

is no consensus regarding the management of tumors at the limit of resectability which remains controversial, as stated in the American College of Chest Physician Guidelines [40]. Studies are expected to clarify this topic and improve the survival of stage III patients. The gold standard for the management of these patients remains the multimodal treatment combining chemotherapy with or without neoadjuvant radiotherapy and radical surgery, followed or not by adjuvant treatment.

\section{References}

1. Silvestri GA, Jett J (2005) Bronchogenic carcinoma. Murray and nadel's textbook of respiratory medicine. Philadelphia: Elsevier saunders (4th ed.): 1371.

2. Lee S, Khan I, Upadhyay S, Lewanski C, Falk S, et al. (2012) First-line erlotinib in patients with advanced non-small-cell lung cancer unsuitable for chemotherapy (TOPICAL): A double-blind, placebo-controlled, phase 3 trial Lancet Oncol 13: 1161-1170.

3. Granetzny A, Striehn E, Bosse U, Wagner W, Koch O, et al. (2003) A phase II single-institution study of neoadjuvant stage IIIA/B chemotherapy and radiochemotherapy in non-small cell lung cancer. Ann Thorac Surg 75: 11071112.

4. Stamatis G, Djuric D, Eberhardt W (2002) Postoperative morbidity and mortality after induction chemoradiotherapy for locally advanced lung cancer: An analysis of 350 operated patients. Eur J Cardiothorac Surg 22: 292-297.

5. NSCLC Meta-analysis Collaborative Group (2014) Preoperative chemotherapy for non-small-cell lung cancer: A systematic review and meta-analysis of individual participant data. Lancet 383: 1561-1571.

6. Jemal A, Bray F, Center MM, Ferlay J, Ward E, et al. (2011) Global cancer statistics. CA Cancer J Clin 61: 69-90.

7. Gadgeel SM, Ramalingam SS, Kalemkerian GP (2012) Treatment of lung cancer. Radiol Clin North Am 50: 961-974.

8. NSCLC Meta-analyses Collaborative Group (2010) Adjuvant chemotherapy, with or without postoperative radiotherapy, in operable non-small-cell lung cancer: Two meta-analyses of individual patient data. Lancet 375: 1267-1277.

9. Detterbeck FC, Lewis SZ, Diekemper R (2013) Executive summary: Diagnosis and management of lung cancer, 3rd ed: American college of chest physicians evidence-based clinical practice guidelines. Chest 143: 7S-37S

10. NSCLC Collaborative Group (2010) Chemotherapy and supportive care versus supportive care alone for advanced non small-cell lung cancer. Cochrane Database Syst Rev 12: CD007309.

11. Perkins P, Kemp BL, Putnam JB Jr, Cox JD (1997) Pretreatment characteristics of carcinoid tumors of the lung which predict aggressive behavior. J Clin Onco 20: $285-288$.

12. Rena O, Oliaro A, Cavallo A, Filosso PL, Donati G, et al. (2002) Stage I nonsmall cell lung carcinoma: Really an early stage?. Eur J Cardiothorac Surg 21: 514-519.

13. Port JL, Kent MS, Korst RJ, Libby D, Pasmantier M, et al. (2003) Tumor size predicts survival within stage IA non-small cell lung cancer. Chest 124: 18281833.

14. Morgensztern D, Waqar S, Subramanian J, Gao F, Trinkaus K, et al. (2012) Prognostic significance of tumor size in patients with stage III non-small-cell lung cancer: A surveillance, epidemiology, and end results (SEER) survey from 1998 to 2003. J Thorac Oncol 7: 1479-1484

15. Voltolini L, Rapicetta C, Luzzi L, Ghiribelli C, Ligabue T, et al. (2006) Lung cancer with chest wall involvement: Predictive factors of long-term survival after surgical resection. Lung Cancer 52: 359-364.

16. Martini N, Yellin A, Ginsberg RJ, Bains MS, Burt ME, et al. (1994) Management of non-small cell lung cancer with direct mediastinal involvement. Ann Thorac Surg 58: 1447-1451.

17. Inoue H, Shohtsu A, Koide S, Ogawa J, Inoue H (1990) Resection of superior vena cava for primary lung cancers: 5 years' survival. Ann Thorac Surg 50: 661-662.

18. Fukuse T, Wada H, Hitomi S (1997) Extended operation for non-small cell lung cancer invading great vessels and left atrium. Eur J Cardiothorac Surg 11: 664 669.

19. Spaggiari L, Rusca M, Carbognani P, Solli P (1998) Hemivertebrectomy for apical chest tumors: Is the risk justified by the outcome?. Ann Thorac Surg 65 1516-1517.

20. Velenz VE (1997) Lymphe node type as a prognostic factor for survival in T2 N1 M0 non-small cell lung carcinoma. Ann Thorac Surg 63: 1436-1439.

21. Martini N (1992) Survival after resection of stage II non-small cell lung cancer Ann Thorac Surg 54: 460-463.

22. Marra A, Hillejan L, Zaboura G, Fujimoto T, Greschuchna D, et al. (2003) Pathologic N1 non-small cell lung cancer: Correlation between pattern of lymphatic spread and prognosis. J Thorac Cardiovasc Surg 125: 543-553.

23. Casali C, Stefani A, Natali P, Rossi G, Morandi U (2005) Prognostic factors in surgically resected N2 non-small cell lung cancer: The importance of patterns of mediastinal lymph nodes metastases. Eur J Cardiothorac Surg 28: 33-38.

24. Sakao Y, Okumura S, Mun M, Uehara H, Ishikawa Y, et al. (2010) Prognostic heterogeneity in multilevel N2 non-small cell lung cancer patients: Importance of lymphadenopathy and occult intrapulmonary metastases. Ann Thorac Surg 89: 1060-106.

25. Rami-Porta R, Crowley JJ, Goldstraw P (2009) The revised TNM staging system for lung cancer. Ann Thorac Cardiovasc Surg 15: 4-9.

26. Okamoto T, Maruyama R, Shoji F, Asoh H, Ikeda J, et al. (2005) Long term survivors in stage IV non- small cell lung cancer. Lung Cancer 47: 85-91.

27. Mountain CF (1997) Revisions in the international systeme for staging lung cancer. Chest 111: 1710-1717.

28. Albin KS, Crowley JJ, LeBlanc M, Livingston RB (1991) Survival determinjants in extensive-stage-non-small cell lung cancer: The southwest Oncology Group experience. J Clin Oncol 9: 1618-1626.

29. Billing PS, Miller DL, Allen MS, Deschamps C, Trastek VF, et al. (2001) Surgical treatment of primary lung cancer with synchronous brain metastases. J Thorac Cardiovasc Surg 122: 548-553.

30. Jacot W, Quantin X, Boher JM, Andre F, Moreau L, et al. (2001) Brain metastases at the time of presentation of non-small cell lung cancer: A multicentric AERIO analysis of prognostic factors. Brit J Cancer 84: 903-909.

31. Salah S, Tanvetyanon T, Abbasi S (2011) Metastatectomy for axtra-crania extra-adrenal non-small cell lung cancer solitary metastases systematic review and analysis of reported cases. Lung Cancer 75: 9-14.

32. Verhagen AF, Tavilla G, van de Wal HJ, Cox AL, Lacquet LK (1994) Multiple primary lung cancers. Thorac Cardiovasc Surg 42: 40-44.

33. Gridelli C, Gallo C, Shepherd FA, Illiano A, Piantedosi F, et al. (2003) Gemcitabine plus vinorelbine compared with cisplatine plus vinorelbine or cisplatine plus gemcitabine for advanced non-small-cell lung cancer: A phase III Trial of the italian EMVIN investigators and the national cancer institute of Canada clinical trials group. J Clin Oncol 21: 3025-3034.

34. Grivaux M, Zureik M, Marsal L, Asselain B, Peureux M, et al. (2009) Five-year survival of primary bronchial cancers in general hospitals. Rev Mal Respir 26 $37-44$.

35. Berghmans T, Lafitte JJ, Giner V, Berchier MC, Scherpereel A, et al. (2012) Final results and pharmacoeconomic analysis of a trial comparing two neoadjuvant chemotherapy (CT) regimens followed by surgery in patients with resectable non-small cell lung cancer (NSCLC): A phase II randomized study by the european lung cancer working party. Lung Cancer 77: 605-610.

36. Kim AW, Liptay MJ, Bonomi P, Warren WH, Basu S, et al. (2011) Neoadjuvan chemoradiation for clinically advanced non-small cell lung cancer: an analysis of 233 patients. Ann Thorac Surg 92: 233-243.

37. Depierre A, Milleron B, Moro-Sibilot D, Chevret S, Quoix E, et al. (2002) Preoperative chemotherapy followed by surgery compared with primary surgery in resectable stage I (except T1N0), II, and IIla non-small-cell lung cancer. J Clin Oncol 20: 247-253.

38. Ripley RT, Rusch VW (2013) Role of induction therapy surgical resection of non-small cell lung cancer after induction therapy. Thorac Surg Clin 23: 273 285

39. Song WA, Zhou NK, Wang W (2010) Survival benefit of neoadjuvant chemotherapy in non-small cell lung cancer: An updated meta-analysis of 13 randomized control trials. J Thorac Oncol 5: 510-516.

40. Ramnath N, Dilling TJ, Harris LJ, Kim AW, Michaud GC, et al. (2013) Treatment of stage III non-small cell lung cancer: Diagnosis and management of lung cancer, 3rd ed: American college of chest physicians evidence-based clinical 
Citation: Hazem Z, Ahmed AB, Abderrahmen A, Mahdi A, Sonia O, et al. (2018) Neoadjuvant Chemotherapy in Lung Cancer: Our Results after Twelve Years of Surgical Resection. J Pulm Respir Med 8: 463. doi: 10.4172/2161-105X.1000463

practice guidelines. Chest 143: e314S-40S.

41. Lim E, Harris G, Patel A, Adachi I, Edmonds L, et al. (2009) Preoperative versus postoperative chemotherapy in patients with resectable non-small cell lung cancer: systematic review and indirect comparison meta-analysis of randomized trials. J Thorac Oncol 4: 1380-1388.

42. Porte HL, Metois DG, Leroy X, Conti M, Gosselin B, et al. (2000) Surgical treatment of primary sarcoma of the lung. Eur J Cardiothorac Surg 18: 136-142.

43. Hirsch FR, Spreafico A, Novello S (2008) The prognostic and predictive role of histology in advanced non-small cell lung cancer: A literature review. J Thorac Oncol 3: 1468-1481.

44. Hellmann MD, Chaft JE, William N, Rusch V, Pisters K (2014) Pathological response after neoadjuvant chemotherapy in resectable non-small-cell lung cancers: proposal for the use of major pathological response as a surrogate endpoint. Lancet Oncol 15: 42-50.

45. Luzzi L, Voltolini L, Campione A, Paladini P, Ghiribelli C, et al. (2003) Pneumonectomy vs lobectomy in the treatment of pathologic N1 NSCLC: Could the type of surgical resection dictate survival?. J Cardiovasc Surg 44: 119-123.
46. Okada M, Nishio W, Sakamoto T, Uchino K, Yuki T, et al. (2005) Effect of tumor size on prognosis in patients with non-small cell lung cancer: The role of segmentectomy as a type of lesser resection. J Thorac Cardiovasc Surg 129: 87-93.

47. Wu YI, Huang SY, Yang XN, Ou W (2002) A randomized trial of systemic nodal dissection in resectable non-small cell lung cancer. Lung Cancer 36: 1-6.

48. Venuta F, Anile M, Diso D, Ibrahim M, De Giacomo T, et al. (2007) Operative complications and early mortality after induction therapy for lung cancer. Eur $\mathrm{J}$ Cardiothoracic Surg 31: 714-717.

49. D'Amato TA, Ashrafi AS, Schuchert MJ, Alshehab DS, Seely AJ, et al. (2009) Risk of pneumonectomy after induction therapy for locally advanced non-small cell lung cancer. Ann Thorac Surg 88: 1079-1085. 